\title{
Giant non-adiabatic effects in layer metals: Raman spectra of intercalated graphite explained
}

\author{
A. Marco Saitta, Michele Lazzeri, Matteo Calandra, and Francesco Mauri \\ IMPMC, Universités Paris 6 et 7, CNRS, IPGP, 140 rue de Lourmel, 75015 Paris, France
}

(Dated: October 29, 2018)

\begin{abstract}
The occurrence of non-adiabatic effects in the vibrational properties of metals have been predicted since the 60's, but hardly confirmed experimentally. We report the first fully ab initio calculations of non-adiabatic frequencies of a number of layer and conventional metals. We suggest that non-adiabatic effects can be a feature of the vibrational Raman spectra of any bulk metal, and show that they are spectacularly large (up to $30 \%$ of the phonon frequencies) in the case of layer metals, such as superconducting $\mathrm{MgB}, \mathrm{CaC}_{6}$ and other graphite intercalated compounds. We develop a framework capable to estimate the electron momentum-relaxation time of a given system, and thus its degree of non-adiabaticity, in terms of the experimentally observed frequencies and linewidths.
\end{abstract}

PACS numbers: 63.20.dk,71.15.-m,63.20.kd,74.70.Ad

The adiabatic Born-Oppenheimer approximation (ABOA) is the state of the art in first-principles calculations of vibrational properties in solids. In metals, even if in principle unjustified, this approximation generally leads to phonon dispersions in very good agreement with experimental data [1]. Indeed violations of the ABOA are hardly visible in solids. Engelsberg and Schrieffer suggested [2] that non-adiabatic (NA) effects could lead to a significant renormalization of zone-center optical phonon frequencies. An intense effort have been devoted to detect measurable NA effects in metals, mainly by Raman spectroscopy [3, 4, 5], however the detected NA phonon-frequencies-renormalizations are typically smaller than some percent of the adiabatic phonon frequency.

Recently it has been shown that NA effects are crucial to interpret the dependence of Raman spectra on doping [6, 7] in graphene and on doping [8, 9] and on diameter and temperature [10] in nanotubes. However, despite being a central issue for the physics of graphene-based systems, the NA phonon frequency shift $\Delta \omega$ (the difference between adiabatic and NA phonon frequencies), although measurable, is less than $1 \%$ of the adiabatic phonon-frequency. Moreover, these systems have lower dimensionality and a peculiar electronic structure, it is then unclear whether sizeable NA effects can actually be observed in truly three-dimensional bulk metals.

Layer metallic materials, such as Graphite Intercalated Compounds (GICs), are three dimensional (3D) metals possessing a considerable anisotropy along one direction. Variation of the interlayer distance by intercalation or applied pressure allows to bridge the gap between two-dimensional monolayer systems (such as graphene) and truly three-dimensional systems [11]. Thus these metals are ideal to judge the role of reduced dimensionality in determining non-adiabatic effects. $\mathrm{CaC}_{6}$ is also an $11.5 \mathrm{~K}$ superconductor[12] with an intermediate electron-phonon coupling $\lambda \approx 0.83$ [13]. Most interestingly, the recent measurement of $\mathrm{CaC}_{6}$ Raman spectrum [14] shows that the phonon frequencies related to in-plane $\mathrm{C}$ vibrations are almost $80 \mathrm{~cm}^{-1}$ larger than those obtained from density-functional theory (DFT) adiabatic calculations. This result is puzzling since in graphite the adiabatic result for the $\mathrm{E}_{2 g}$ phonon frequency is $1577 \mathrm{~cm}^{-1}$, in excellent agreement with the experimental value of $1582 \mathrm{~cm}^{-1}$.

In this Letter we develop a first-principles theoretical framework to calculate the magnitude of NA effects on zonecenter optical-phonons in metals. We identify the general conditions for having sizeable adiabatic effects in 3D bulk metals and analyze the experimental constraints that can hinder the observation of NA effects. We demonstrate that the occurrence of NA effects is not limited to reduced dimensionality but it is a general properties of metals. We apply our approach to GICs, $\mathrm{MgB}_{2}$ and a few bulk metals, finding giant NA effects. To the best of our knowledge, our work is the first systematic implementation and study of non-adiabatic effects in a first principles framework.

Non adiabatic effects due to the treatment of the electronphonon coupling in the Migdal approximation (neglecting of vertex corrections in the adiabatic limit) are usually of the order $\sqrt{m / M}$, where $m$ and $M$ are the electronic and ionic mass respectively, and thus generally very small. Engelsberg and Schrieffer [2] showed that NA renormalization of adiabatic phonon frequencies, a larger effect, unrelated to the neglecting of vertex corrections, and not of the order of $\sqrt{m / M}$, can be observed if the following two conditions are satisfied:

$$
\begin{aligned}
\left|\mathbf{q} \cdot \mathbf{v}_{\mathbf{F}}\right| & \ll \omega \\
\hbar \omega & \gg \sigma
\end{aligned}
$$

where $\mathbf{v}_{\mathbf{F}}$ is the Fermi velocity, $\mathbf{q}$ is the phonon wavevector, $\omega$ is the phonon frequency, $\sigma=\hbar / \tau$ and $\tau$ is the electron momentum-relaxation time (Drude) of the electrons near the Fermi surface due to all possible momentum-exchange scattering mechanisms.

The first condition is verified for optical phonons of small wavevector whose phase velocity is larger than the electronic Fermi velocity. Even if in principle Eq. (1) can be fulfilled in any system, provided $\mathbf{q}$ is small enough, in practice the penetration length of the laser light limits the smallest exchanged $\mathbf{q}$ in Raman experiments. The fact that Eq. (1) is experimentally difficult to satisfy in 3D metals explains why the observation of NA effects in these systems has been somehow disappointing.

The second condition states that the electron momentum- 
relaxation time must be much larger than the phonon period. This implies that the electron band-population dynamic is too slow to follow the atomic motion and thus the dynamic is nonadiabatic [7]. Eq. (2) is usually verified in pure and well crystallized samples at low temperature. Thus Eq. (1) is the main limitation to the observation of NA effects.

This limitation can be circumvented considering system having small $\mathbf{v}_{F}$ along certain directions. For example layer metals are usually characterized by a small $\mathbf{v}_{\mathbf{F}}$ component perpendicular to the layers. Since the samples are usually cleaved parallel to the layers and Raman experiments are performed perpendicularly to the freshly cleaved surface, the scalar product $\mathbf{q} \cdot \mathbf{v}_{F}$ is small. It is instead also evident that condition (1) is easily verified in 2D or 1D systems, since Raman experiments are performed with an incident light of wavevector $\mathbf{q}$ perpendicular to the sample spatial dimension(s) and, thus, to $\mathbf{v}_{F}$. In fact, NA effects have been consistently observed in doped graphene and nanotubes in the last few years.

NA effects can be taken into account by applying time-dependent perturbation theory to DFT. Neglecting the electron-momentum scattering rate $(\sigma=0)$, the dynamical matrix $(\mathcal{D})$ for a phonon at $\mathbf{q}=\mathbf{0}(\boldsymbol{\Gamma})$ and the first-order variation of the electronic charge density $(\Delta n)$ are [15]:

$$
\begin{aligned}
\mathcal{D}_{\boldsymbol{\Gamma}}(\omega) & =\frac{2}{N_{\mathbf{k}}} \sum_{\mathbf{k} n, m \neq n} \frac{\left|D_{\mathbf{k} m, \mathbf{k} n}\right|^{2}\left[f_{\mathbf{k} m}-f_{\mathbf{k} n}\right]}{\epsilon_{\mathbf{k} m}-\epsilon_{\mathbf{k} n}+\hbar \omega} \\
& +\int n(\mathbf{r}) \Delta^{2} V^{b}(\mathbf{r}) d \mathbf{r} \\
& -\int \Delta n_{\boldsymbol{\Gamma}}(\mathbf{r}, \omega) K\left(\mathbf{r}, \mathbf{r}^{\prime}\right) \Delta n_{\boldsymbol{\Gamma}}(\mathbf{r}, \omega) d \mathbf{r} d \mathbf{r}^{\prime} \\
\Delta n_{\boldsymbol{\Gamma}}(\mathbf{r}, \omega) & =\frac{2}{N_{\mathbf{k}}} \sum_{\mathbf{k} n, m \neq n} \frac{\langle\mathbf{k} n \mid \mathbf{r}\rangle\langle\mathbf{r} \mid \mathbf{k} m\rangle D_{\mathbf{k} m, \mathbf{k} n}\left[f_{\mathbf{k} m}-f_{\mathbf{k} n}\right]}{\epsilon_{\mathbf{k} m}-\epsilon_{\mathbf{k} n}+\hbar \omega}
\end{aligned}
$$

where $n(\mathbf{r})$ is the charge density, the sum is performed on $N_{\mathbf{k}}$ k-points, $D_{\mathbf{k} m, \mathbf{k} n}=\left\langle\mathbf{k} m\left|\Delta V^{s c}\right| \mathbf{k} n\right\rangle$ is the deformation potential proportional to the electron-phonon matrix element, $\Delta V^{s c}$ and $\Delta^{2} V^{b}$ are, respectively, the first derivative of the Kohn-Sham potential and the second derivative of the bare (purely ionic) potential with respect to the phonon displacement. The kernel $K\left(\mathbf{r}, \mathbf{r}^{\prime}\right)$ is the second functional derivative of the Hartree exchange and correlation potential respect to the densities at $\mathbf{r}$ and $\mathbf{r}^{\prime}$. Finally $f_{\mathbf{k} n}$ is the Fermi function for a Bloch state $|\mathbf{k} n\rangle$ having band energy $\epsilon_{\mathbf{k} n}$. The NA frequencies has to be computed self-consistenty from $\omega^{N A}=\sqrt{\mathcal{D}_{\Gamma}\left(\omega^{N A}\right) / M}$. Since in Eqs. (3) and (4) only interband transitions contribute, if $\hbar \omega^{N A} \ll\left|\epsilon_{\mathbf{k} e}-\epsilon_{\mathbf{k} o}\right|$, i.e. if the phonon energy is much smaller than the direct gap between empty $\left(\epsilon_{\mathbf{k} e}\right)$ and occupied $\left(\epsilon_{\mathbf{k} o}\right)$ states, $\mathcal{D}_{\boldsymbol{\Gamma}}\left(\omega^{N A}\right) \simeq \mathcal{D}_{\boldsymbol{\Gamma}}(0)$. We verified numerically that in all systems considered in the present study this approximation applies and that the results obtained with $\mathcal{D}_{\boldsymbol{\Gamma}}\left(\omega^{N A}\right)$ and $\mathcal{D}_{\boldsymbol{\Gamma}}(0)$ are indistinguishable.

The adiabatic frequency $\omega^{A}$ is as usual calculated within static perturbation theory, as in Ref. [1]. Note that $\omega^{A} \neq$ $\sqrt{\mathcal{D}_{\Gamma}(0) / M}$ since in the adiabatic case the intraband $(m=n)$

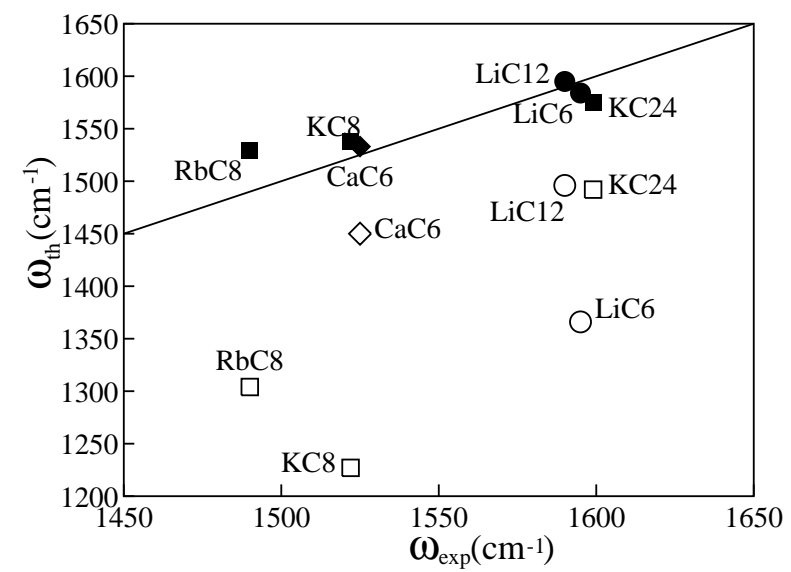

FIG. 1: Calculated adiabatic (empty symbols) and non-adiabatic (filled symbols) $\mathrm{E}_{2 g}$ phonon frequencies of several GICs, compared to the available experimental data. The full line represents the experimental values.

term is present [6] and $\Delta \omega=\omega^{N A}-\omega^{A}$ is:

$$
\hbar \Delta \omega \simeq \frac{1}{N_{\mathbf{k}}} \sum_{\mathbf{k} n} \frac{\hbar\left|D_{\mathbf{k} n, \mathbf{k} n}\right|^{2}}{M \omega^{A}} \delta\left(\epsilon_{F}-\epsilon_{\mathbf{k} n}\right)=n\left(\epsilon_{F}\right) \overline{g^{2}\left(\epsilon_{F}\right)},
$$

where $n\left(\epsilon_{F}\right)$ is the density of states at the Fermi level, $g^{2}\left(\epsilon_{f}\right)=\hbar\left|D_{\mathbf{k} n, \mathbf{k} n}\right|^{2} / 2 M \omega^{A}$ is the square electron-phonon matrix element due to intraband transitions, and $\overline{g^{2}\left(\epsilon_{F}\right)}$ is the average of $g^{2}\left(\epsilon_{f}\right)$ over the Fermi surface.

We apply our first principles approach to obtain $\mathrm{E}_{2 g}$ nonadiabatic phonon frequencies at $\boldsymbol{\Gamma}$ for a number of GICs, for $\mathrm{MgB}_{2}$ and for bulk $h c p \mathrm{Ti}$ (see footnote [16] for computational details). The results are illustrated in Fig. 1 for GICs and are collected in Tab. I for all the systems. In general huge $\Delta \omega$ values $\left(>60 \mathrm{~cm}^{-1}\right)$ are found in all layer compounds. The most spectacular non-adiabatic effects are found in $\mathrm{KC}_{8}\left(\Delta \omega=310 \mathrm{~cm}^{-1}, 20 \%\right.$ of $\left.\omega^{A}\right)$ and in $\operatorname{MgB}_{2}(\Delta \omega=$ $230 \mathrm{~cm}^{-1}, 30 \%$ of $\left.\omega^{A}\right)$. Even in bulk $T i$ we find a significant shift, $\Delta \omega=12 \mathrm{~cm}^{-1}$, (more than $8 \%$ of $\omega^{A}$ ). From Fig. 1 it is evident that experimental Raman data [17, 18, 19, 20, 21] in all GICs are in much closer agreement with $\omega^{N A}$ than with $\omega^{A}$, whereas in $\mathrm{MgB}_{2}$ Raman data lie in between the two theoretical frequencies. In Ti, contrary to the layer metals, the observed Raman frequencies [22] are much closer to the adiabatic value.

To further refine our theoretical model, we now compute the phonon frequency in presence of a finite value of the electronmomentum relaxation rate $\sigma, \omega^{\sigma}=\omega^{A}+\Delta \omega^{\sigma} . \Delta \omega^{\sigma}$ can be obtained using electron and hole Green functions dressed by the interaction (electron-electron, electron-phonon, and electron-defect) in the calculation of the phonon self-energy and of the dynamical matrix [3, 23, 24]. Assuming $i) \sigma$ independent of energy and ii) $n(\epsilon)$ and $g\left(\epsilon_{f}\right)$ constants for bands 
within $\epsilon_{F} \pm \sigma$, Refs. [3, 23, 24] obtained:

$$
\hbar \Delta \omega^{\sigma} \simeq \hbar \Delta \omega \frac{\left(\hbar \omega^{A}\right)^{2}}{\left(\hbar \omega^{A}\right)^{2}+\sigma^{2}} .
$$

Note that when $\hbar \omega^{A} \gg \sigma, \Delta \omega^{\sigma} \simeq \Delta \omega$ and the experimentally observed phonon frequency is $\omega^{N A}$, i.e. the system is in the purely NA regime. On the contrary, for $\hbar \omega^{A} \ll \sigma$, $\Delta \omega^{\sigma}=0$ and the measured phonon frequency is $\omega^{A}$, i.e. the system is completely adiabatic.

The linewidth of an optical phonon mode at $\boldsymbol{\Gamma}$ is also affected by the presence of a finite momentum-relaxation rate. The decay of a phonon into non-interacting (undressed) electron-hole pairs $(\sigma=0)$ is forbidden for a zone-center optical mode if the direct gap is larger than the phonon energy $\left(\hbar \omega \ll\left|\epsilon_{\mathbf{k} e}-\epsilon_{\mathbf{k} o}\right|\right)$. This condition being verified in all layer systems considered here, thus a zero linewidth should be measured [25, 26]. However in all stage-1 GICs and in $M g B_{2}$, very large linewidths, hardly explainable in term of anharmonicities, are measured. Analogously to the calculation of $\omega^{\sigma}$, a finite momentum-scattering rate $\sigma$ can be considered in the evaluation of the imaginary part of the phonon self-energy [3, 23, 24, 27]. This leads to the following expression for the phonon full linewidth at half maximum due to the phonon decay in dressed electron-hole pairs $\left(\gamma_{\sigma}^{E P C}\right)$ :

$$
\frac{\gamma_{\sigma}^{E P C}}{2}=\hbar \Delta \omega \frac{\sigma \hbar \omega^{A}}{\left(\hbar \omega^{A}\right)^{2}+\sigma^{2}} .
$$

Eq. (6) can be used to extract $\sigma$. Setting $\Delta \omega^{\sigma}=\omega_{\exp }-\omega^{A}$, we obtain $\sigma$ from the inversion of Eq. (6). Then $\sigma$ is inserted in Eq. (7) to determine $\gamma_{\sigma}^{E P C}$ and the results are reported in Tab. [1 The comparison between $\gamma_{\sigma}^{E P C}$ and the experimental Raman linewidths $\Gamma_{\text {exp }}$ is shown in Fig. 2, The agreement is overall very good, except for $\mathrm{LiC}_{6}$ and $\mathrm{RbC}_{8}$. Note that the experimental linewidth includes all sort of broadening (including inhomogeneous effects), while $\gamma_{\sigma}^{E P C}$ includes only the phonon decay into dressed electron-hole pairs. Thus when $\Gamma_{\exp } \approx \gamma_{\sigma}^{E P C}$ it means that the dominant broadening mechanism is the latter one. This is the case in $\mathrm{MgB}_{2}$ [27, 28], and in most GICs. Moreover, by comparing these results with the universal curves of Eqs. (6) and (7) (Fig. 3), we deduce that our estimate of the momentum-relaxation time is a good indicator of the degree of non-adiabaticity.

As an example, our results quantitatively indicate that $M g B_{2}$, although being characterized by a huge $\Delta \omega$, has a smaller $\Delta \omega^{\sigma}$ and a relatively short momentum-relaxation time. It is thus a mostly adiabatic system, even though $\Delta \omega^{\sigma}$ is still very large. On the contrary, all GICs have relatively small $\sigma$ 's, falling in the left part of the universal curves of Fig. 3. and are thus mostly non-adiabatic. The result on $h c p$ titanium actually indicates that all metals might have NA $\Gamma$ frequencies significantly different from the adiabatic ones, but that they cannot be experimentally observed by Raman because the condition (1) is not verified in experiments. In other words, non-adiabaticity is not a unique property of low-dimensional or layer systems, and can occur even in perfectly conventional

\begin{tabular}{|c|c|c|c|c|c|c|c|}
\hline \hline & $\omega^{A}$ & $\omega^{N A}$ & $\omega_{\exp }$ & $\Delta \omega$ & $\sigma$ & $\gamma_{\sigma}^{E P C}$ & $\Gamma_{\text {exp }}$ \\
\hline$L i C_{6}$ & 1362 & 1580 & 1595 & 218 & 0 & 0 & 70 \\
$L i C_{12}$ & 1492 & 1591 & 1590 & 99 & 151 & 20 & 66 \\
\hline$K C_{8}$ & 1223 & 1534 & 1522 & 311 & 245 & 120 & 157 \\
$K C_{24}$ & 1488 & 1571 & 1599 & 83 & 0 & 0 & 26 \\
\hline$R b C_{8}(4 \mathrm{~K})$ & 1300 & 1525 & 1490 & 225 & 558 & 163 & 100 \\
$R b C_{8}(77 \mathrm{~K})$ & 1300 & 1525 & 1480 & 225 & 650 & 180 & 120 \\
\hline$C a C_{6}(5 \mathrm{~K})$ & 1446 & 1529 & 1525 & 83 & 325 & 36 & 71 \\
$C a C_{6}(300 \mathrm{~K})$ & 1446 & 1529 & 1511 & 83 & 761 & 68 & 111 \\
\hline$S r C_{6}$ & 1459 & 1530 & - & 71 & - & - & - \\
\hline$B a C_{6}$ & 1462 & 1521 & - & 59 & - & - & - \\
\hline \hline$M g B_{2}(21 \mathrm{~K})$ & 538 & 761 & 600 & 224 & 867 & 199 & 197 \\
\hline \hline$M g$ & 122 & 123 & 122.5 & 1 & N/A & N/A & N/A \\
\hline \hline$T i$ & 139 & 151 & 141 & 12 & N/A & N/A & N/A \\
\hline \hline
\end{tabular}

TABLE I: Calculated adiabatic and non-adiabatic $\mathrm{E}_{2 g}$ phonon frequencies, experimental frequencies, theoretical frequency difference, calculated $\sigma$, calculated EPC linewidth, experimental linewidth of several GICs and of $M g B_{2}$. Experimental data are from Refs. [14, 17, 18, 19, 20, 21, 29, 30] Symbols indicate systems for which experimental data are available, and are the same as in the figures. For $M g$ and $T i$ the determination of $\sigma$ from our theory is not applicable. We note that since our best NA frequency of graphite is $1578 \mathrm{~cm}^{-1}$, we have upshifted by $4 \mathrm{~cm}^{-1}$ all the adiabatic/NA frequencies of GICs.

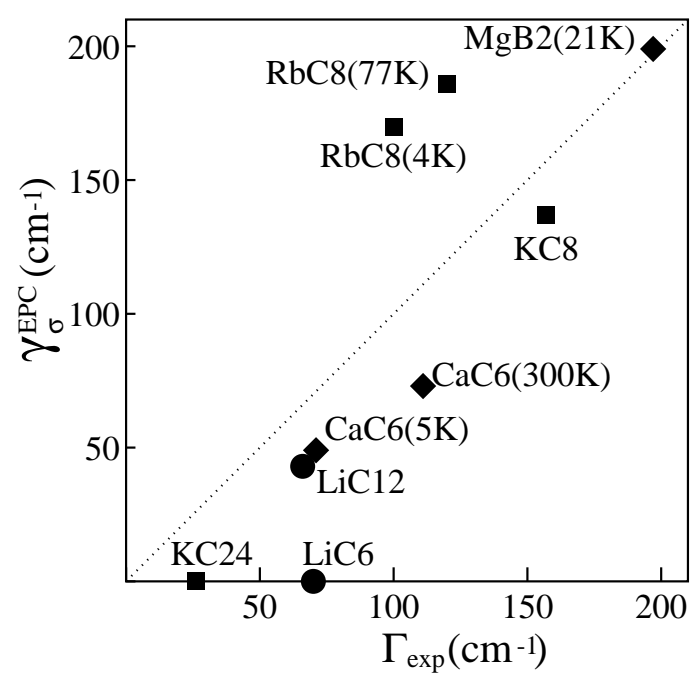

FIG. 2: Calculated EPC linewidth (Eq. 7) with respect to the observed experimental ones.

metals. Interestingly, despite the structural similarity, in bulk $M g$ no NA effect exists.

In conclusion, we provide a quantitative first-principles theoretical framework to explain the difference between the reported experimental $\mathrm{E}_{2 g}$ mode frequencies, and the (ordinary) adiabatic calculated ones in several relevant layer metals. We have shown that giant non-adiabatic effects occur in these systems. Moreover, NA effects are in principle very relevant even in bulk metals, although difficult to measure. Finally, we have 


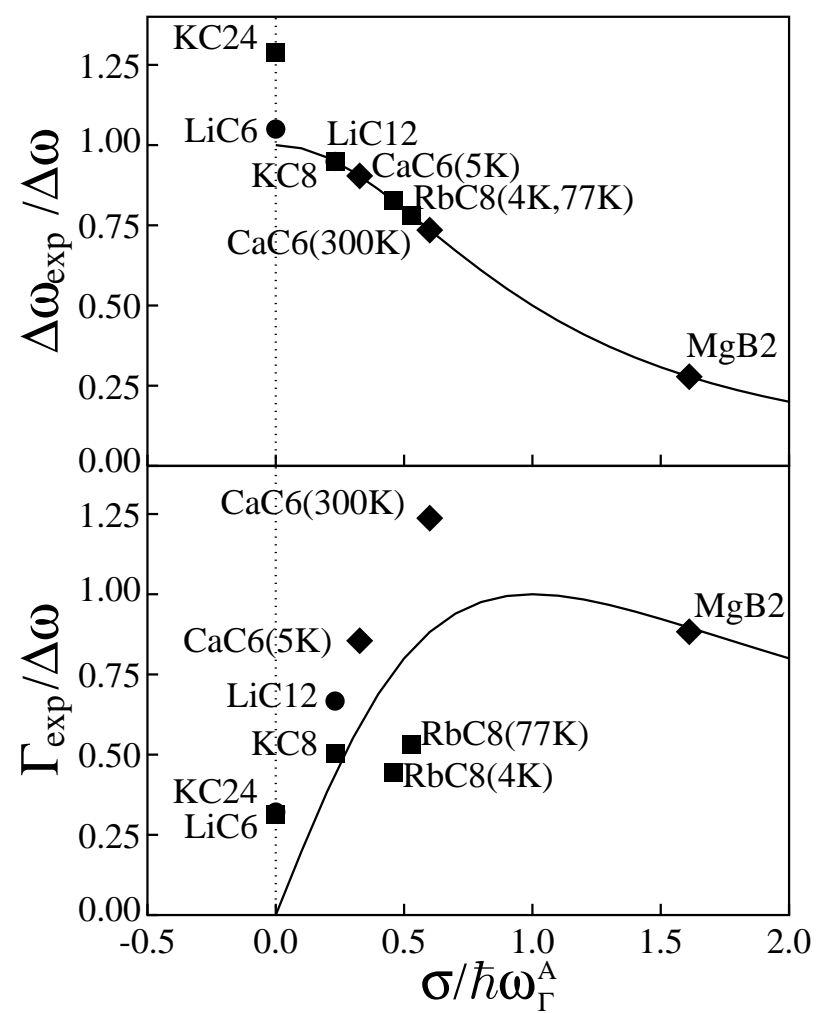

FIG. 3: Upper panel: $\hbar \Delta \omega_{\sigma} / \hbar \Delta \omega$ (Eq. 6, solid line), compared to measured frequencies (symbols). Lower panel: $\gamma_{\sigma}^{E P C} / \hbar \Delta \omega$ (Eq.7. solid line), and measured linewidths (FWHM).

shown that the electron momentum-relaxation time can be extracted from Raman peak positions and linewidths and is a good indicator of the degree of non-adiabaticity of the system.

Calculations have been performed at the IDRIS French National Computational Facility under the projet CP9-71387.

[1] S. Baroni, S. de Gironcoli, A. D. Corso, and P. Giannozzi, Rev. Mod. Phys. 73, 515 (2001).

[2] S. Engelsberg and J. Schieffer, Phys. Rev. 131, 993 (1963).

[3] E. Maksimov and S. Shulga, Solid State Comm. 97, 553 (1995).

[4] Y. S. Ponosov, G. A. Bolotin, C. Thomsen, and M. Cardona, Phys. Stat. Sol. (b) 208, 257 (1998).

[5] G. Bolotin, Y. Kuz'min, Y. Knyazev, Y. Ponosov, and C. Thomsen, Phys. Sol. State 43, 1801 (2001).

[6] M. Lazzeri and F. Mauri, Phys. Rev. Lett. 97, 266407 (2006).

[7] S. Pisana, M. Lazzeri, C. Casiraghi, K. S. Novoselov, A. K. Geim, A. C. Ferrari, and F. Mauri, Nature Materials 6, 198 (2007).

[8] N. Caudal, A. M. Saitta, M. Lazzeri, and F. Mauri, Phys. Rev. B 75, 115423 (2007).

[9] A. Das, A. K. Sood, A. Govindaraj, A. M. Saitta, M. Lazzeri, F. Mauri, and C. N. Rao, Phys. Rev. Lett. 99, 136803 (2007).
[10] S. Piscanec, M. Lazzeri, J. Robertson, A. C. Ferrari, and F. Mauri, Phys. Rev. B 75, 035427 (2007).

[11] M. Calandra and F. Mauri, Phys. Rev. B 74, 094507 (2006).

[12] T. E. Weller, M. Ellerby, S. S. Saxena, R. P. Smith, and N. T. Skipper, Nature Physics 1, 39 (2005).

[13] M. Calandra and F. Mauri, Phys. Rev. Lett. 71, 205210 (2005).

[14] J. Hlinka, I. G. andJ. Pokorny, C. Hérold, N. Emery, J. Marêché, and P. Lagrange, Phys. Rev. B 76, 144512 (2007).

[15] For the sake of simplicity we consider atoms having identical masses $\mathrm{M}$.

[16] We focus on several stable lithium, potassium, rubidium, calcium, barium and strontium GICs, at stages 1 or 2 of intercalation, as well as on $M g B_{2}$. In particular, we study the stage1 GICs $\mathrm{LiC}_{6}, \mathrm{KC}_{8}, \mathrm{RbC}_{8}, \mathrm{CaC}_{6}, \mathrm{BaC}_{6}$, and $\mathrm{SrC}_{6}$, and the stage-2 $\mathrm{LiC}_{12}$ and $\mathrm{KC}_{24}$. Our first-principles calculations are based on DFT, within the plane-wave (PW)/pseudopotential scheme implemented in the Quantum-ESPRESSO code [31]. We adopt a Perdew-Burke-Erzherhof gradient corrected functional, and ultrasoft pseudopotentials to describe the different atom species. The PW kinetic energy cutoffs are comprised between 35 and $60 \mathrm{Ry}$, according to the system. We adopted the available experimental structural parameters for GIC's containing $L i$ [18], $K$ and $R b$ [17], $C a, B a$, and $S r$ [11], and for $M g B_{2}$ [11]. In the case of $K C_{8}$ and $R b C_{8}$ the phonon calculations were performed by adopting a $A \alpha A \alpha$ stacking since the energy differences with respect to the $A \alpha A \beta A \gamma A \delta$ stacking are negligible. Analogously, in the case of stage- $2 K C_{24}$ a slightly different unit cell was adopted for the phonon calculations rather than the $K_{2} C_{48}$ unit cell suggested in [17]. Integrations in the BZ have been performed by using regular grids from $(8 \times 8 \times 4)$ to $(64 \times 64 \times 24) \mathbf{k}$ points. We use a MethfesselPaxton electronic smearing of $0.01 \mathrm{Ry}$. For a faster convergence with $\mathbf{k}$ points sampling, The numerical results reported in the paper are obtained with $\mathcal{D}_{\boldsymbol{\Gamma}}(0)$, since the k-points-sampling convergence of this quantity is faster than that of $\mathcal{D}_{\Gamma}\left(\omega^{N A}\right)$.

[17] M. Dresselhaus and G. Dresselhaus, Adv. Phys. 51, 1 (2002).

[18] D. Guérard and C. Hérold, Carbon 13, 337 (1975).

[19] P. Eklund, G. Dresselhaus, M. Dresselhaus, and J. Fischer, Phys. Rev. B 16, 3330 (1977).

[20] P. Eklund, G. Dresselhaus, M. Dresselhaus, and J. Fischer, Phys. Rev. B 21, 4705 (1980).

[21] G. Doll, P. Eklund, and J. Fischer, Phys. Rev. B 36, 4940 (1987).

[22] C. Stassis, D. Arch, B. Harmon, and N. Wakabayashi, Phys. Rev. B 19, 181 (1979).

[23] A. Zawadowski and M. Cardona, Phys. Rev. B 42, 10732 (1990).

[24] F. Marsiglio, R. Akis, and J. P. Carbotte, Phys. Rev. B 45, 9865 (1992).

[25] O. Jepsen, I. I. Mazin, A. I. Liechtenstein, O. K. Andersen, , and C. O. Rodriguez, Phys. Rev. B 51, 3961 (1995).

[26] M. Calandra and F. Mauri, Phys. Rev. B 71, 064501 (2005).

[27] E. Cappelluti, Phys. Rev. B 73, 140505 (2006).

[28] M. Calandra, M. Lazzeri, and F. Mauri, Physica C 456, 38 (2007).

[29] J. Quilty, S. Lee, A. Yamamoto, and S. Tajima, Phys. Rev. Lett. 88, 087001 (2002).

[30] G. Doll, M. Yang, and P. Eklund, Phys. Rev. B 35, 9790 (1987).

[31] S. Baroni, A. Dal Corso, S. de Gironcoli, P. Giannozzi, C. Cavazzoni, G. Ballabio, S. Scandolo, G. Chiarotti, P. Focher, A. Pasquarello, et al., www . quantum-espresso.org. 